\title{
Termination-of-resuscitation rule for emergency department physicians treating out-of-hospital cardiac arrest patients: an observational cohort study
}

Yoshikazu Goto ${ }^{1 *}$, Tetsuo Maeda ${ }^{1}$ and Yumiko Nakatsu Goto ${ }^{2}$

\begin{abstract}
Introduction: The 2010 cardiopulmonary resuscitation guidelines recommend emergency medical services (EMS) personnel consider prehospital termination-of-resuscitation (TOR) rules for out-of-hospital cardiac arrest (OHCA) following basic life support and/or advanced life support efforts in the field. However, the rate of implementation of international TOR rules is still low. Here, we aimed to develop and validate a new TOR rule for emergency department physicians to replace the international TOR rules for EMS personnel in the field. This rule aims to guide physicians in deciding whether to withhold further resuscitation attempts or terminate on-going resuscitation immediately after patient arrival.
\end{abstract}

Methods: We analyzed data prospectively collected in a nationwide Utstein-style Japanese database between 2005 and 2009, from 495,607 adult patients with OHCA. Patients were divided into development $(n=390,577)$ and validation ( $n=105,030)$ groups. The main outcome measures were specificity, positive predictive value (PPV), and area under the receiver operating characteristic (ROC) curve for the newly developed TOR rule.

Results: We developed a new TOR rule that includes 3 criteria based on the results of multivariate logistic regression analysis for predicting a 1-month death after OHCA: no prehospital return of spontaneous circulation (adjusted odds ratio [OR], 25.8; 95\% confidence interval [CI], 24.7-26.9), unshockable initial rhythm (adjusted OR, 2.76; 95\% Cl, 2.54-3.01), and unwitnessed by bystanders (adjusted OR, 2.18; 95\% Cl, 2.09-2.28). The specificity, PPV, and area under the ROC curve for this new TOR rule for predicting 1-month death in the validation group were 0.903 (95\% Cl, 0.894-0.911), 0.993 (95\% Cl, 0.992-0.993), and 0.874 (95\% Cl, 0.872-0.876), respectively.

Conclusions: We developed and validated a new TOR rule for emergency department physicians consisting of 3 prehospital variables (no prehospital ROSC, unshockable initial rhythm, and unwitnessed by bystanders) that is a $>99 \%$ predictor of very poor outcome. However, the implementation of this new rule in other countries or EMS systems requires further validation studies.

\section{Introduction}

Out-of-hospital cardiac arrest (OHCA) has a poor prognosis and is a leading cause of death in the developed world. The incidence of OHCA treated by emergency medical services (EMS) personnel has been estimated to be approximately 275,000 persons per year, with a survival rate of $10.7 \%$ for all initial rhythm in Europe [1] and

\footnotetext{
* Correspondence: gotoyosh@med.kanazawa-u.ac.jp

${ }^{1}$ Section of Emergency Medicine, Kanazawa University Hospital, 13-1

Takaramachi, Kanazawa 920-8641, Japan

Full list of author information is available at the end of the article
}

approximately 300,000 persons per year with a survival rate of 9.6\% in United States [2]. Despite decades of research, the survival rates after OHCA have remained virtually unchanged in the past three decades [3,4].

The 2010 American Heart Association (AHA) Guidelines for Cardiopulmonary Resuscitation and Emergency Cardiovascular Care [5] recommend that EMS personnel consider prehospital termination of resuscitation (TOR) for patients with OHCA following basic life support (BLS) and/or advanced life support (ALS) efforts in the field. The prehospital BLS TOR rule with three 
criteria--unwitnessed by EMS personnel, no shock given and no prehospital return of spontaneous circulation (ROSC)--for EMS personnel was originally developed in Toronto by Verbeek et al. [6] and has been validated worldwide [7-10]. The authors of the original BLS TOR rule derived an ALS TOR rule with two additional criteria [11]. These TOR rules for OHCA have been implemented to better utilize hospital healthcare resources, reduce the number of attendant hazards to EMS personnel and the considerable associated financial expense, and increase the availability of care and transport for other patients [6-12].

The decision to terminate resuscitation efforts is fraught with ethical and legal considerations [5]. Therefore, any guidelines for TOR in the field must be highly reliable, accurate and legally defensible [9]. Currently, the rate of implementation of the TOR rules is low [13-16]. Different TOR rules, other than the aforementioned BLS and ALS TOR rules, should be established with reliability in different EMS systems. EMS personnel in Japan, however, are not legally allowed to terminate resuscitation for OHCA patients in the field; therefore, almost all OHCA patients are transported to a hospital, regardless of whether resuscitation is successful. Any TOR rules in the prehospital settings are thus not legally implemented in Japan. Therefore, a new TOR rule for emergency department physicians is required to replace the international TOR rules for EMS personnel in the field to allow for better utilization of hospital healthcare resources.

In this study, we aimed to establish and validate a new TOR rule for emergency department physicians that would allow them to decide whether to withhold further resuscitation attempts or terminate ongoing resuscitation immediately after patient arrival. Moreover, we validated the BLS TOR rule to compare the relevance of a new TOR rule.

\section{Materials and methods}

\section{Study design and data source}

The present study was a nationwide population-based observational study in all adult patients ages 18 years and older for whom resuscitation had been attempted after OHCA in Japan from 1 January 2005 to 31 December 2009. Cardiac arrest was defined as the cessation of cardiac mechanical activities confirmed by the absence of signs of circulation [17]. The cause of arrest was presumed to be of cardiac origin unless evidence suggested external causes (trauma, hanging, drowning, drug overdose and asphyxia), respiratory diseases, cerebrovascular diseases, malignant tumors or any other noncardiac causes. Attribution of noncardiac or cardiac etiology was made by the physicians in charge in collaboration with the EMS personnel. This study was approved by the Ethical Committee of Kanazawa
University. The requirement for written informed consent was waived.

\section{Emergency medical services system in Japan}

Japan has approximately 127 million residents in an area of $378,000 \mathrm{~km}^{2}$, approximately two-thirds of which is uninhabited mountainous terrain [18]. Details on the Japanese EMS system have been described elsewhere $[18,19]$. Briefly, municipal governments provide EMS through approximately 800 fire stations with dispatch centers. The Fire and Disaster Management Agency (FDMA) of Japan supervises only the EMS system nationwide, and individual EMS systems are operated by each local fire station. Generally, an ambulance crew consists of three EMS staff members, including at least one emergency lifesaving technician (ELST). ELSTs are allowed to use several resuscitation methods, including semiautomated external defibrillators, insertion of an adjunct airway, insertion of a peripheral intravenous line and administration of Ringer lactate solution. Since July 2004, only specially trained ELSTs have been permitted to insert a tracheal tube, and since April 2006, they have been permitted to administer intravenous epinephrine in the field under online physician instruction. Since October 2006, all EMS providers have performed cardiopulmonary resuscitation (CPR) according to the Japanese CPR guidelines [17], which are based on the 2005 AHA Guidelines for Cardiopulmonary Resuscitation and Emergency Cardiovascular Care [20]. As EMS personnel in Japan are legally prohibited from terminating resuscitation in the field, most OHCA patients undergo CPR by EMS providers and are transported to hospitals, except in cases where fatality is certain [18]. The length of the on-scene effort by EMS personnel is not predetermined before transport is initiated. Epinephrine use is implemented according to the FDMA resuscitation guidelines for ELSTs $[17,21]$. The permitted dosage of epinephrine is $1 \mathrm{mg}$ per attempt, and repeated doses may be administered under the physician's instruction.

\section{Data collection and quality control}

The FDMA launched a prospective population-based observational study of all OHCA victims who received EMS in Japan [18] since January 2005. EMS personnel at each center, using an Utstein-style template, recorded data for OHCA victims with the cooperation of the physician in charge [22]. All data were transferred and stored in a nationwide database developed by the FDMA for public use. For this study, we analyzed this anonymous database with the permission of the FDMA. The main items included in the database are patient sex, age, etiology of arrest (presumed cardiac origin or not), bystander witness status, bystander CPR with or without automated external defibrillator use, initial identified 
cardiac rhythm, bystander category (that is, if there was a bystander, then whether the bystander was a layperson or EMS personnel), whether epinephrine was administered, whether advanced airway management (including endotracheal tube, laryngeal mask airway and esophagealtracheal tube) were used, whether ROSC was achieved before arrival at the hospital, time of the emergency call, time of vehicle arrival at the scene, time of ROSC, time of vehicle arrival at the hospital, one-month survival and neurological outcome at one month after cardiac arrest. Neurological outcome was defined on the basis of the Cerebral Performance Category (CPC) scale: category 1, good cerebral performance; category 2, moderate cerebral disability; category 3, severe cerebral disability; category 4, coma or vegetative state; and category 5 , death [22]. This CPC categorization was determined by the physicians in charge. The call-to-response time was calculated as the time from the emergency call to the time of vehicle arrival at the scene. The call-to-hospital arrival time was calculated as the time from the emergency call to the time of vehicle arrival at the hospital.

\section{Main outcome measures}

The main outcome measures were specificity, positive predictive value (PPV) and area under the receiver operating characteristic (ROC) curve for the newly developed TOR rule for emergency department physicians. Secondary outcome measures were those related to the BLS TOR rule.

\section{Statistical analysis}

Kolmogorov-Smirnov and Lilliefors tests were performed to evaluate the distributions of continuous variables, and we found that all continuous variables were not normally distributed (all $P<0.01$ ). Therefore, the Wilcoxon and Kruskal-Wallis tests for continuous variables and the $x^{2}$ test for categorical variables were performed to compare the basic characteristics of patients between the development and validation groups. Multivariate logistic regression analyses including 11 variables were performed to assess the association between prehospital variables and onemonth death or unfavorable neurological outcome in the development group. Continuous variables are expressed as medians (interquartile range (IQR)) [1st to 3rd quartiles], and categorical variables are expressed as percentages. As an estimate of effect size and variability, we report odds ratios (ORs) with 95\% confidence intervals (CIs). All statistical analyses were performed with the JMP version 10 statistical discovery software package (SAS Institute Inc, Cary, NC, USA). All tests were two-tailed, and $P<0.05$ was considered statistically significant.

\section{Results}

During the five-year study period, 547,218 patients were documented in the database. From among these patients,
495,607 (90.6\%) were eligible for enrollment in this study. Figure 1 shows a flow diagram depicting the inclusion and exclusion criteria for patients in the present study. Patients were divided into a development group (2005 to $2008 ; n=390,577$ ) and a validation group (2009; $n=105,030)$. The characteristics of all patients and the results of analyses of the two groups are shown in Table 1. Because of the large size of the study population, several significant differences were noted in baseline characteristics between the two groups; however, sizeable differences were less frequent, except for the ratio of bystander CPR. Overall rates of one-month survival and CPC categories 1 and 2 were $3.93 \%$ and $1.69 \%$, respectively. The validation group had significantly higher one-month survival and one-month CPC categories 1 and 2 rates than the development group (survival: $4.34 \%$ vs. $3.81 \%$; CPC categories 1 and 2: $2.04 \%$ vs. $1.60 \%$; all $P<0.0001)$.

\section{Development of a new termination-of-resuscitation rule for emergency department physicians}

The results of multivariate logistic regression analyses, including 11 variables to determine the factors associated with one-month death and CPC categories 3 to 5, are shown in Table 2. No prehospital ROSC had the highest adjusted OR for one-month death (adjusted OR, 25.8 ; $95 \%$ CI, 24.7 to 26.9 ) followed by unshockable initial rhythm (adjusted OR, 2.76; 95\% CI, 2.54 to 3.01) and unwitnessed by bystanders (adjusted OR, 2.18; 95\% CI, 2.09 to 2.28 ). We selected three prehospital variables that had adjusted ORs greater than 2.0 for predicting one-month death as predictors for one-month outcomes. Therefore, these three variables were incorporated into the new TOR rule, meaning that TOR was newly defined as appropriate in cases fulfilling all three criteria: no prehospital ROSC, unshockable initial rhythm and unwitnessed cardiac arrest by bystanders. No prehospital ROSC was also strongly associated with one-month CPC categories 3 to 5 with an adjusted OR of 38.4 (95\% CI, 35.9 to 41.0$)$.

\section{Analysis of derivation and validation datasets of the new termination-of-resuscitation rule}

Table 3 shows the results of the analysis of derivation (development group) and validation (validation group) data sets of the new TOR rule for predicting one-month death. The rates of patients who fulfilled all three criteria in the development and validation groups were $57.1 \%$ and $57.3 \%$, respectively, both of which would be considered futile. The new TOR rule showed a specificity of 0.875 (95\% CI, 0.870 to 0.881 ), PPV of 0.992 (95\% CI, 0.991 to 0.992 ) and area under the ROC curve of 0.851 ( $95 \%$ CI, 0.850 to 0.852 ) in the development group. The specificity, PPV and area under the ROC curve for the 


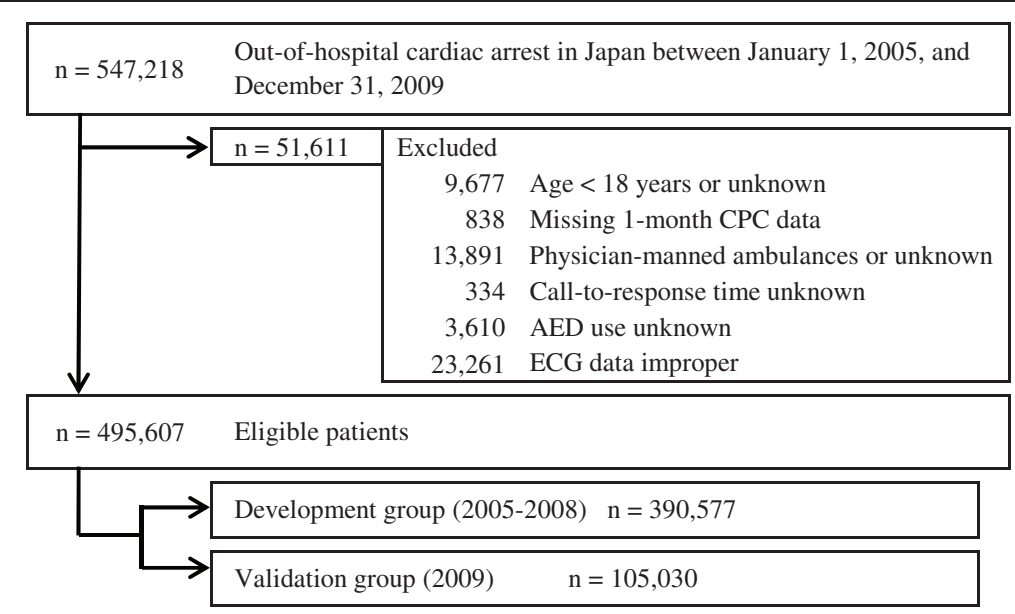

Figure 1 Study profile and selection of patient population. AED, automated external defibrillator; $C P C$, cerebral performance category; ECG, electrocardiogram.

validation group were 0.903 ( $95 \% \mathrm{CI}, 0.894$ to 0.911 ), 0.993 (95\% CI, 0.992 to 0.993 ) and 0.874 (95\% CI, 0.872 to 0.876 ), respectively. Table 4 shows the results of the analysis of derivation and validation data sets of the new TOR rule for predicting a one-month unfavorable neurological outcome. The new TOR rule showed a specificity of 0.939 ( $95 \%$ CI, 0.933 to 0.945 ), PPV of 0.998 (95\% CI, 0.998 to 0.999 ) and area under the ROC curve of 0.922 ( $95 \%$ CI, 0.921 to 0.923 ) in the development group. The specificity, PPV and area under the ROC curve for the validation group were 0.966 ( $95 \%$ CI, 0.958 to 0.973 ), 0.999 (95\% CI, 0.999 to 0.999$)$ and 0.942 (95\% CI, 0.941 to 0.944$)$, respectively.
Analysis of validation dataset of the basic life support termination-of-resuscitation rule

Table 5 shows the results of analysis of the 2009 validation data set $(N=105,030)$ of the BLS TOR rule for predicting one-month death. The rate of patients who fulfilled all three criteria of the BLS rule in the validation group was $81.6 \%$, which would be considered futile. The BLS TOR rule showed a specificity of 0.779 (95\% CI, 0.767 to 0.791 ), PPV of 0.988 (95\% CI, 0.988 to 0.989 ) and area under the ROC curve of 0.811 (95\% CI, 0.809 to 0.813 ). Table 6 shows the results of the analysis of the validation data set of the BLS TOR for predicting onemonth unfavorable neurological outcomes. The BLS

Table 1 Baseline characteristics of the study patients ${ }^{a}$

\begin{tabular}{|c|c|c|c|c|}
\hline Characteristics & $\begin{array}{l}\text { All patients } \\
(N=495,607,100 \%)\end{array}$ & $\begin{array}{l}\text { Development group, } \\
2005 \text { to } 2008 \\
(n=390,577,78.8 \%)\end{array}$ & $\begin{array}{l}\text { Validation group, } 2009 \\
(n=105,030,21.2 \%)\end{array}$ & $P$-value \\
\hline Age, years & 76 (64 to 84$)$ & 76 (64 to 84$)$ & 77 (65 to 85$)$ & $<0.0001$ \\
\hline Males & $290,712(58.7 \%)$ & $229,822(58.8 \%)$ & $60,890(58.0 \%)$ & $<0.0001$ \\
\hline Witnessed cardiac arrest & $188,471(38.0 \%)$ & $147,997(37.9 \%)$ & $40,474(38.5 \%)$ & $<0.0001$ \\
\hline Cardiac arrest witnessed by EMS personnel & $22,722(4.6 \%)$ & $17,514(4.5 \%)$ & $5,208(5.0 \%)$ & $<0.0001$ \\
\hline Bystander CPR & 202,827 (40.9\%) & $151,712(38.8 \%)$ & $51,115(48.7 \%)$ & $<0.0001$ \\
\hline Presumed cardiac etiology & $276,183(55.7 \%)$ & $216,242(55.4 \%)$ & $59,941(57.0 \%)$ & $<0.0001$ \\
\hline Shockable initial cardiac rhythm & $38,388(7.75 \%)$ & $30,154(7.7 \%)$ & $8,234(7.8 \%)$ & 0.1992 \\
\hline Prehospital AED administration & $53,427(10.8 \%)$ & $42,216(10.8 \%)$ & $11,211(10.7 \%)$ & 0.212 \\
\hline Call-to-response time, minutes & $9(7$ to 11$)$ & $6(5$ to 9$)$ & 7 (5 to 9) & $<0.0001$ \\
\hline Call-to-hospital arrival time, minutes & 29 (23 to 36$)$ & 29 (23 to 36$)$ & 30 (24 to 37$)$ & $<0.0001$ \\
\hline Prehospital ROSC & $25,254(5.1 \%)$ & $18,835(4.8 \%)$ & $6,419(6.1 \%)$ & $<0.0001$ \\
\hline \multicolumn{5}{|l|}{ One-month outcome after cardiac arrest } \\
\hline Survival & $19,453(3.93 \%)$ & $14,898(3.81 \%)$ & $4,555(4.34 \%)$ & $<0.0001$ \\
\hline Favorable neurological outcome (CPC category 1 or 2 ) & 8,391 (1.69\%) & $6,244(1.60 \%)$ & $2,147(2.04 \%)$ & $<0.0001$ \\
\hline
\end{tabular}

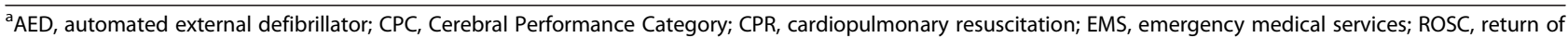
spontaneous circulation. Values are reported as either number of patients (\%) or medians (interquartile range [1st to 3rd quartiles]). 
Table 2 Results of univariate and multivariate logistic regression analyses for factors associated with one-month outcomes $^{\text {a }}$

\begin{tabular}{|c|c|c|c|c|}
\hline \multirow[t]{2}{*}{ Variables } & \multicolumn{2}{|l|}{ Death } & \multicolumn{2}{|c|}{ CPC categories 3 to 5} \\
\hline & Crude OR $(95 \% \mathrm{Cl})$ & Adjusted OR $(95 \% \mathrm{Cl})$ & Crude OR $(95 \% \mathrm{Cl})$ & Adjusted OR $(95 \% \mathrm{Cl})$ \\
\hline $\mathrm{Age}^{\mathrm{b}}$ & $1.02(1.02$ to 1.02$)$ & $1.01(1.01$ to 1.01$)$ & $1.03(1.03$ to 1.03$)$ & $1.03(1.02$ to 1.03$)$ \\
\hline Gender (male) & $1.40(1.35$ to 1.45$)$ & 1.00 (0.96 to 1.05$)$ & 0.53 (0.50 to 0.56$)$ & 0.90 (0.84 to 0.96$)$ \\
\hline Unwitnessed by bystanders & 4.70 (4.54 to 4.88$)$ & 2.18 (2.09 to 2.28 ) & 7.04 (6.61 to 7.50$)$ & 2.01 (1.87 to 2.17 ) \\
\hline Unwitnessed by EMS personnel & 3.51 (3.33 to 3.68 ) & $1.69(1.59$ to 1.81$)$ & 5.10 (4.77 to 5.44$)$ & 2.68 (2.46 to 2.93 ) \\
\hline No bystander CPR & $1.13(1.10$ to 1.17$)$ & $1.01(0.97$ to 1.06$)$ & $1.29(1.23$ to 1.36$)$ & $1.11(1.04$ to 1.18$)$ \\
\hline Noncardiac etiology & $1.38(1.34$ to 1.43$)$ & 0.93 (0.89 to 0.97 ) & $3.00(2.83$ to 3.19$)$ & $1.84(1.71$ to 1.99$)$ \\
\hline Unshockable initial rhythm & 10.4 (10.0 to 10.8$)$ & 2.76 (2.54 to 3.01$)$ & $21.6(20.5$ to 22.7$)$ & 3.38 (2.98 to 3.84$)$ \\
\hline No prehospital AED administration & 7.50 (7.25 to 7.76$)$ & 1.54 (1.41 to 1.67$)$ & $15.2(14.4$ to 16.0$)$ & $1.42(1.24$ to 1.61$)$ \\
\hline No prehospital ROSC & 43.4 (41.8 to 45.0$)$ & 25.8 (24.7 to 26.9$)$ & 80.2 (75.5 to 85.2$)$ & $38.4(35.9$ to 41.0$)$ \\
\hline Call-to-response time ${ }^{\mathrm{b}}$ & $1.10(1.10$ to 1.11$)$ & $1.05(1.04$ to 1.06$)$ & $1.13(1.12$ to 1.14$)$ & $1.05(1.04$ to 1.06$)$ \\
\hline Call-to-hospital arrival time ${ }^{b}$ & $1.02(1.01$ to 1.02$)$ & $1.03(1.02$ to 1.03$)$ & $1.02(1.02$ to 1.02$)$ & $1.02(1.02$ to 1.03$)$ \\
\hline
\end{tabular}

${ }^{a} \mathrm{AED}$, automated external defibrillator; $\mathrm{Cl}$, confidence interval; $\mathrm{CPC}$, cerebral performance category; CPR, cardiopulmonary resuscitation; EMS, emergency medical services; OR, odds ratio; ROSC, return of spontaneous circulation. ${ }^{b}$ Adjusted odds ratios are reported for unit odds.

TOR rule showed a specificity of 0.928 ( $95 \% \mathrm{CI}, 0.917$ to 0.938), PPV of 0.998 ( $95 \% \mathrm{CI}, 0.997$ to 0.998 ) and area under the ROC curve of 0.880 ( $95 \% \mathrm{CI}, 0.871$ to 0.889 ).

\section{Discussion}

We developed and validated a new rule to guide physicians in deciding whether to terminate resuscitation after OHCA immediately after patient arrival at the emergency department. A new TOR rule was defined to fulfill the following three criteria: no prehospital ROSC, unshockable initial rhythm and unwitnessed cardiac arrest by bystanders. Figure 2 shows a flowchart algorithm of how the new TOR rule should be applied. If a patient with OHCA fulfills all three criteria immediately after patient arrival at the emergency department, the physician in charge should consider terminating resuscitation before performing ALS. Our present results demonstrate that the new TOR rule has high specificity, PPV and area under the ROC curve for predicting one-month outcomes, although our TOR rule could not fully predict one-month death. We validated the BLS TOR rule using the validation data set to compare the performance of the new TOR rule. Our new TOR rule had higher specificity, PPV and area under the ROC curve than the BLS TOR rule for predicting one-month outcomes. This finding implies that the new rule is preferable to the BLS TOR rule in Japan. Unlike the international TOR rules for EMS personnel in the field, the new TOR rule presents no burden to EMS personnel for determining the futility of CPR.

TOR in the field is a crucial issue for both patients with OHCA and healthcare staff, including EMS personnel. Although TOR clinical prediction rules can minimize costs and lead to better use of EMS resources [7], ethical issues around TOR remain controversial. In 1990, an objective criterion for medical futility was defined for interventions and drug therapy imparting a less than $1 \%$ chance of survival [23], and this level remains a basis for current futility research [5]. Our newly

Table 3 Performance of the new termination-of-resuscitation rule for predicting one-month death ${ }^{\mathrm{a}}$

\begin{tabular}{|c|c|c|c|c|}
\hline \multirow{3}{*}{ Variables } & \multicolumn{2}{|c|}{ Development group $(N=390,577)$} & \multicolumn{2}{|c|}{ Validation group $(N=105,030)$} \\
\hline & Fulfilled $3 / 3$ criteria & Did not fulfill criteria & Fulfilled $3 / 3$ criteria & Did not fulfill criteria \\
\hline & $(n=223,023,57.1 \%)$ & $(n=167,554,42.9 \%)$ & $\overline{(n=60,205,57.3 \%)}$ & $(n=44,825,42.7 \%)$ \\
\hline Death, $n$ & 221,167 & 154,512 & 59,763 & 40,712 \\
\hline Survival, $n$ & 1,856 & 13,042 & 442 & 4,113 \\
\hline Sensitivity (95\% CI) & \multicolumn{2}{|c|}{0.589 (0.587 to 0.590$)$} & \multicolumn{2}{|c|}{0.595 (0.592 to 0.598$)$} \\
\hline Specificity $(95 \% \mathrm{Cl})$ & \multicolumn{2}{|c|}{0.875 (0.870 to 0.881$)$} & \multicolumn{2}{|c|}{0.903 (0.894 to 0.911$)$} \\
\hline PPV $(95 \%$ Cl) & \multicolumn{2}{|c|}{0.992 (0.991 to 0.992) } & \multicolumn{2}{|c|}{0.993 (0.992 to 0.993 ) } \\
\hline NPV (95\% Cl) & \multicolumn{2}{|c|}{0.078 (0.077 to 0.079$)$} & \multicolumn{2}{|c|}{0.092 (0.089 to 0.095$)$} \\
\hline Area under the ROC curve $(95 \% \mathrm{Cl})$ & \multicolumn{2}{|c|}{0.851 (0.850 to 0.852 ) } & \multicolumn{2}{|c|}{0.874 (0.872 to 0.876$)$} \\
\hline
\end{tabular}

${ }^{a} \mathrm{Cl}$, confidence interval; PPV, positive predictive value; NPV, negative predictive value; $\mathrm{ROC}$, receiver operating characteristic. 


\begin{tabular}{|c|c|c|c|c|}
\hline \multirow{3}{*}{ Variables } & \multicolumn{2}{|c|}{ Development group $(N=390,577)$} & \multicolumn{2}{|c|}{ Validation group $(N=105,030)$} \\
\hline & Fulfilled $3 / 3$ criteria & Did not fulfill criteria & Fulfilled $3 / 3$ criteria & Did not fulfill criteria \\
\hline & $(n=223,023,57.1 \%)$ & $(n=167,554,42.9 \%)$ & $(n=60,205,57.3 \%)$ & $(n=44,825,42.7 \%)$ \\
\hline Unfavorable (CPC categories 3 to 5 ), $n$ & 222,642 & 161,691 & 60,132 & 42,751 \\
\hline Favorable (CPC categories 1 and 2), $n$ & 381 & 5,863 & 73 & 2,074 \\
\hline Sensitivity (95\% Cl) & \multicolumn{2}{|c|}{0.579 (0.578 to 0.581$)$} & \multicolumn{2}{|c|}{0.585 (0.582 to 0.588$)$} \\
\hline Specificity (95\% Cl) & \multicolumn{2}{|c|}{0.939 (0.933 to 0.945$)$} & \multicolumn{2}{|c|}{0.966 (0.958 to 0.973$)$} \\
\hline PPV $(95 \%$ Cl) & \multicolumn{2}{|c|}{0.998 (0.998 to 0.999$)$} & \multicolumn{2}{|c|}{0.999 (0.999 to 0.999) } \\
\hline NPV (95\% Cl) & \multicolumn{2}{|c|}{0.035 (0.034 to 0.036$)$} & \multicolumn{2}{|c|}{$0.046(0.044$ to 0.048$)$} \\
\hline Area under the ROC curve $(95 \% \mathrm{Cl})$ & \multicolumn{2}{|c|}{0.922 (0.921 to 0.923$)$} & \multicolumn{2}{|c|}{0.942 (0.941 to 0.944$)$} \\
\hline
\end{tabular}

${ }^{a} \mathrm{CPC}$, Cerebral Performance Category; Cl, confidence interval; PPV, positive predictive value; NPV, negative predictive value; ROC, receiver operating characteristic

established TOR rule showed misclassification rates of less than $1 \%$ for predicting both death $(0.8 \%$ for the development group and $0.7 \%$ for the validation group) and unfavorable outcomes $(0.2 \%$ for the development group and $0.1 \%$ for the validation group) at one month after OHCA.

Kajino et al. [19] recently investigated whether international TOR rules can predict one-month outcomes for selected OHCA patients with presumed cardiac etiologies. They showed that the BLS TOR rule [7] had a PPV of 0.990 (95\% CI, 0.989 to 0.990 ) and a specificity of 0.878 ( $95 \%$ CI, 0.872 to 0.884 ) for one-month death. However, our new TOR rule for OHCA patients with any etiology has a slightly higher PPV and specificity than those in the Kajino et al. study. A possible reason for this difference is the different inclusion and exclusion criteria used between the studies. Moreover, this OHCA registry has been the source of several previous studies [18,24,25].

Both the European Resuscitation Council [26] and the AHA [5] have developed guidelines for the ethical termination of unsuccessful resuscitation to help EMS personnel identify futile resuscitation efforts in the prehospital setting. Despite international TOR guidelines, the estimated rate of adherence to the AHA guidelines at the local level is below 50\% [13]. Sasson et al. [15] identified three distinct groups of stakeholders whose current policies may impede efforts to adopt TOR rules: payers who incentivize transport, legislators who create state mandates for transport and allow only narrow use of do-not-resuscitate orders and communities in which cultural norms are perceived to impede TOR. In a survey of emergency physicians, $92 \%$ of respondents cited fear of litigation as a reason for continuing futile resuscitation efforts in cases of cardiopulmonary arrest [14]. The prevalent "rescue culture" of EMS providers has also been a barrier to the implementation of TOR rules [15]. Furthermore, the optimal duration of CPR prior to terminating resuscitation efforts in the field has not yet been defined $[27,28]$. Therefore, taking these circumstances into consideration, a new TOR rule for emergency department physicians may also serve EMS personnel in the field to minimize costs and better utilize healthcare resources.

New treatments such as hypothermia [29] and extracorporeal CPR [30] for cardiac arrest, as well as improvements in prehospital system factors such as time to start CPR and time to defibrillation [26], may improve outcomes following OHCA. Therefore, our new TOR rule for physicians should be modified periodically with the emergence of new treatments and the evolution of social systems.

Although do-not-resuscitate orders and living wills are generally not used in Japan [19], end-of-life decisions are complex and can be influenced by individual; international; and local cultural, legal, traditional, religious, social and economic factors [26]. Accordingly, the new TOR rule for emergency department physicians should be validated prospectively before implementation. In addition, further discussion of end-of-life decisions and

Table 5 Performance of the basic life support terminationof-resuscitation rule for predicting one-month death ${ }^{\mathrm{a}}$

\begin{tabular}{|c|c|c|}
\hline \multirow[b]{2}{*}{ Validation group } & \multicolumn{2}{|c|}{ BLS TOR rule } \\
\hline & Fulfilled $3 / 3$ criteria & Did not fulfill criteria \\
\hline$(N=105,030)$ & $(n=85,728,81.6 \%)$ & $(n=19,302,18.4 \%)$ \\
\hline Death, $n$ & 84,723 & 15,752 \\
\hline Survival, $n$ & 1,005 & 3,550 \\
\hline Sensitivity (95\% CI) & \multicolumn{2}{|c|}{$0.843(0.841$ to 0.846$)$} \\
\hline Specificity (95\% Cl) & \multicolumn{2}{|c|}{0.779 (0.767 to 0.791$)$} \\
\hline PPV (95\% Cl) & \multicolumn{2}{|c|}{0.988 (0.988 to 0.989$)$} \\
\hline NPV $(95 \%$ Cl) & \multicolumn{2}{|c|}{$0.184(0.179$ to 0.189$)$} \\
\hline $\begin{array}{l}\text { Area under the ROC } \\
\text { curve }(95 \% \mathrm{Cl})\end{array}$ & \multicolumn{2}{|c|}{0.811 (0.809 to 0.813$)$} \\
\hline
\end{tabular}

${ }^{\mathrm{a} B L S}$, basic life support; $\mathrm{Cl}$, confidence interval; NPV, negative predictive value; $\mathrm{PPV}$, positive predictive value; ROC, receiver operating characteristic; TOR, termination of resuscitation. 
Table 6 Performance of the basic life support termination-of-resuscitation rules for predicting onemonth unfavorable neurological outcomes ${ }^{a}$

\begin{tabular}{|c|c|c|}
\hline \multirow[b]{2}{*}{ Validation Group } & \multicolumn{2}{|c|}{ BLS TOR rule } \\
\hline & Fulfilled $3 / 3$ criteria & Did not fulfill criteria \\
\hline$(N=105,030)$ & $\overline{(n=85,728,81.6 \%)}$ & $(n=19,302,18.4 \%)$ \\
\hline $\begin{array}{l}\text { Unfavorable (CPC } \\
\text { categories } 3 \text { to } 5), n\end{array}$ & 85,575 & 17,308 \\
\hline $\begin{array}{l}\text { Favorable (CPC } \\
\text { categories } 1 \text { and 2), } n\end{array}$ & 153 & 1,994 \\
\hline Sensitivity (95\% Cl) & \multicolumn{2}{|c|}{0.832 (0.829 to 0.834 ) } \\
\hline Specificity (95\% Cl) & \multicolumn{2}{|c|}{0.928 (0.917 to 0.938 ) } \\
\hline PPV (95\% CI) & \multicolumn{2}{|c|}{0.998 (0.997 to 0.998) } \\
\hline NPV (95\% Cl) & \multicolumn{2}{|c|}{0.103 (0.099 to 0.108 ) } \\
\hline $\begin{array}{l}\text { Area under the ROC } \\
\text { curve }(95 \% \mathrm{Cl})\end{array}$ & \multicolumn{2}{|c|}{0.880 (0.871 to 0.889 ) } \\
\hline
\end{tabular}

${ }^{\mathrm{a} B L S}$, basic life support; $\mathrm{Cl}$, confidence interval; $\mathrm{CPC}$, cerebral performance category; NPV, negative predictive value; PPV, positive predictive value; $\mathrm{ROC}$, receiver operating characteristic; TOR, termination of resuscitation.

ethical considerations after futile CPR is required, including education and debriefings for healthcare professionals.

\section{Study limitations}

The potential limitations of the current analysis are as follows. First, we did not evaluate detailed in-hospital interventions. We assumed that $\mathrm{OHCA}$ patients received standard ALS according to the Japanese CPR guidelines [17], which were based on the 2005 AHA guidelines [20]. Second, there may be unmeasured confounding factors that could have influenced outcomes. However, the use of uniform data collection on the basis of Utstein-style guidelines for reporting cardiac arrest, the large sample size and a population-based design were intended to minimize these potential sources of bias. Third, the relevance of our results to other communities with different emergency care protocols and different OHCA causes remains unknown. Although TOR outside the hospital is not allowed in some Asian countries [31-33], EMS systems in those countries are different from the Japanese EMS system. Therefore, studies in other countries may be required to validate our results. Fourth, emergency department physicians must pay attention to the use of the new TOR rule for patients with special conditions such as accidental hypothermia. Fifth, emergency department physicians should decide whether further medication is needed, even if patients meet the newly derived TOR rule. Finally, no measure of interrater reliability is obtainable from the data set to guide the determination of the rate of misclassification.

\section{Conclusions}

We have developed and validated a new TOR rule consisting of three prehospital variables (no prehospital ROSC, unshockable initial rhythm and unwitnessed by bystanders) for emergency department physicians. This new TOR rule would offer a rule with a more than $99 \%$ predictor of very poor outcome. However, the implementation of this new rule in other countries or EMS systems, especially in systems with a higher OHCA survival rate than Japan's, requires further validation studies.

\section{Key messages}

- We analyzed prospectively recorded, nationwide, Utstein-style Japanese data over a five-year period

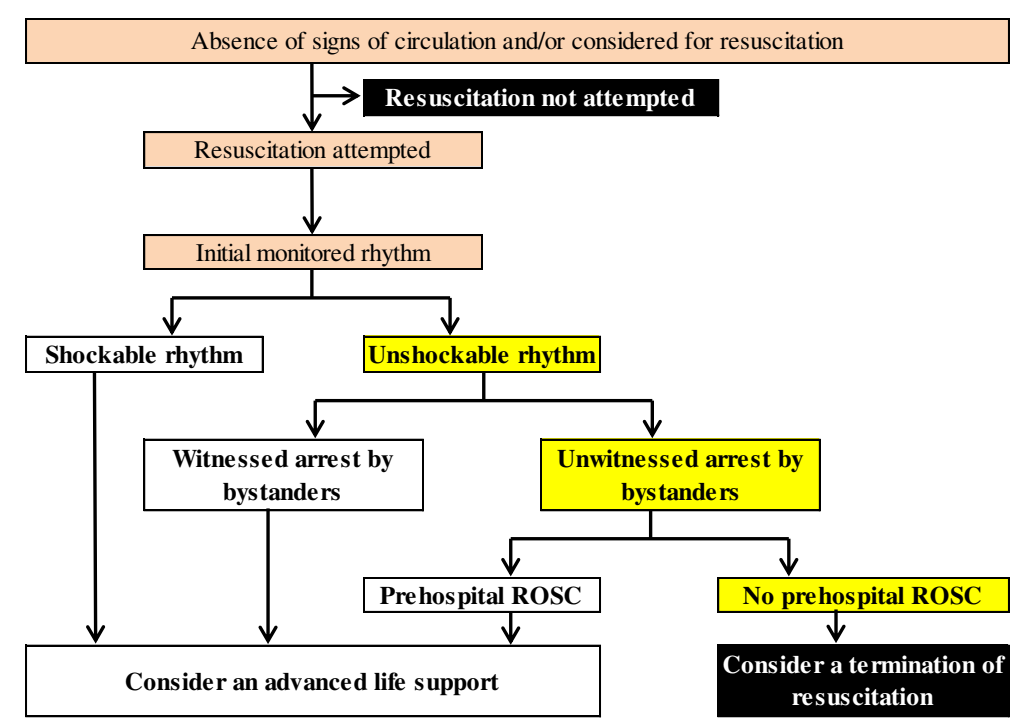

Figure 2 Flowchart algorithm of new termination-of-resuscitation rule for emergency department physicians according to the Utstein template. ROSC, return of spontaneous circulation. 
and developed a new TOR rule, which includes three criteria (no prehospital ROSC, unshockable initial rhythm and unwitnessed by bystanders) for emergency department physicians.

- We validated the new TOR and BLS TOR rules and found that the new TOR rule showed higher specificity, PPV and area under the ROC curve for predicting one-month poor outcomes than those of the BLS TOR rule.

- This new TOR rule would offer a rule that finds a more than $99 \%$ predictor of very poor outcome.

- Further validation studies are required before this new TOR rule can be implemented in other countries or EMS systems.

\begin{abstract}
Abbreviations
AHA: American Heart Association; ALS: Advanced life support; BLS: Basic life support; Cl: Confidence interval; CPC: Cerebral performance category; CPR: Cardiopulmonary resuscitation; ELST: Emergency lifesaving technician; EMS: Emergency Medical Services; FDMA: Fire and Disaster Management Agency; IQR: Interquartile range; OHCA: Out-of-hospital cardiac arrest; OR: Odds ratio; PPV: Positive predictive value; ROC: Receiver operating characteristic; ROSC: Return of spontaneous circulation; TOR: Termination of resuscitation.
\end{abstract}

\section{Competing interests}

The authors declare that they have no competing interests.

\section{Authors' contributions}

YG and TM designed the study. YG, TM and YNG conducted data cleaning. YG and YNG analyzed the data. YG drafted the manuscript. YNG and TM contributed substantially to manuscript revision. YG takes responsibility for the paper as a whole. All authors approved the manuscript before submission. All authors read and approved the final manuscript.

\section{Acknowledgements}

We thank all the EMS personnel and participating physicians in Japan, as well as the FDMA for their generous cooperation in establishing and maintaining the database. This work was supported by grants from the Ministry of Education, Culture, Sports, Science, and Technology (23659253).

\section{Author details}

${ }^{1}$ Section of Emergency Medicine, Kanazawa University Hospital, 13-1 Takaramachi, Kanazawa 920-8641, Japan. ${ }^{2}$ Department of Cardiology, Yawata Medical Center, 12-7 I Yawata, Komatsu 923-8551, Japan.

Received: 26 June 2013 Accepted: 16 September 2013 Published: 13 October 2013

\section{References}

1. Atwood C, Eisenberg MS, Herlitz J, Rea TD: Incidence of EMS-treated outof-hospital cardiac arrest in Europe. Resuscitation 2005, 67:75-80.

2. McNally B, Robb R, Mehta M, Vellano K, Valderrama AL, Yoon PW, Sasson C, Crouch A, Perez AB, Merritt R, Kellermann A: Out-of-hospital cardiac arrest surveillance - to Cardiac Arrest Registry to Enhance Survival (CARES), United States, October 1, 2005 to December 31, 2010. MMWR Surveill Summ 2011, 60:1-19.

3. Nichol G, Thomas E, Callaway CW, Hedges J, Powell JL, Aufderheide TP, Rea T, Lowe R, Brown T, Dreyer J, Davis D, Idris A, Stiell I, Resuscitation Outcomes Consortium Investigators: Regional variation in out-of-hospital cardiac arrest incidence and outcome. JAMA 2008, 300:1423-1431.

4. Sasson C, Rogers MAM, Dahl J, Kellermann AL: Predictors of survival from out-of-hospital cardiac arrest: a systematic review and meta-analysis. Cir Cardiovasc Qual Outcomes 2010, 3:63-81.

5. Morrison LJ, Kierzek G, Diekema DS, Sayre MR, Silvers SM, Idris AH, Mancini ME: Part 3: Ethics. 2010 American Heart Association Guidelines for
Cardiopulmonary Resuscitation and Emergency Cardiovascular Care. Circulation 2010, 122:S665-S675.

6. Verbeek PR, Vermeulen MJ, Ali FH, Messenger DW, Summers J, Morrison LJ: Derivation of a termination-of-resuscitation guideline for emergency medical technicians using automated external defibrillators. Acad Emerg Med 2002, 9:671-678.

7. Morrison LJ, Visentin LM, Kiss A, Theriault R, Eby D, Vermeulen M, Sherbino J, Verbeek PR, for the TOR investigators: Validation of a rule for termination of resuscitation in out-of-hospital cardiac arrest. N Engl J Med 2006, 355:478-487.

8. Sasson C, Hegg AJ, Macy M, Park A, Kellermann A, McNally B, for the CARES Surveillance Group: Prehospital termination of resuscitation in cases of refractory out-of-hospital cardiac arrest. JAMA 2008, 300:1432-1438.

9. Ong MEH, Tan EH, Ng FSP, Yap S, Panchalingham A, Leong BSH, Ong VY, Tiah L, Lim SH, Venkataraman A, for the CARE Study Group: Comparison of termination-of-resuscitation guidelines for out-of-hospital cardiac arrest in Singapore EMS. Resuscitation 2007, 75:244-251.

10. Skrifvars MB, Vayrynen T, Kuisma M, Castren M, Parr MJ, Silfverstople J, Svensson L, Jonsson L, Herlitz J: Comparison of Helsinki and European Resuscitation Council "do not attempt to resuscitate" guidelines, and a termination of resuscitation clinical prediction rule for out-of-hospital cardiac arrest patients found in asystole or pulseless electrical activity. Resuscitation 2010, 81:679-684.

11. Morrison LJ, Verbeek PR, Vermeulen MJ, Kiss A, Allan KS, Nesbitt L, Stiell I: Derivation and evaluation of a termination of resuscitation clinical prediction rule for advanced life support providers. Resuscitation 2007 74:266-275.

12. Morrison LJ, Verbeek PR, Zhan C, Kiss A, Allan KS: Validation of a universal prehospital termination of resuscitation clinical prediction rule for advanced and basic life support providers. Resuscitation 2009, 80:324-328.

13. O'Brien E, Hendricks D, Cone DC: Field termination of resuscitation: analysis of a newly implemented protocol. Prehosp Emerg Care 2008, 12:57-61.

14. Marco CA, Bessman ES, Kelen GD: Ethical issues of cardiopulmonary resuscitation: comparison of emergency physician practices from 1995 to 2007. Acad Emerg Med 2009, 16:270-273.

15. Sasson C, Forman J, Krass D, Macy M, Kellermann AL, McNally BF: A qualitative study to identify barriers to local implementation of prehospital termination of resuscitation protocols. Circ Cardiovasc Qual Outcomes 2009, 2:361-368.

16. Grudzen CR, Hoffman JR, Koenig WJ, Boscardin J, Lorenz KA, Asch SM: The LA story: what happened after a new policy allowing paramedics to forgo resuscitation attempts in prehospital cardiac arrest. Resuscitation 2010, 81:685-690.

17. Japan Resuscitation Council: Japanese Guideline for Emergency Care and Cardiopulmonary Resuscitation. 3rd edition. Tokyo: Health Shupansha; 2007.

18. Kitamura T, Iwami T, Kawamura T, Nagao K, Tanaka H, Hiraide A, for the Implementation Working Group for the All-Japan Utstein Registry of the Fire and Disaster Management Agency: Nationwide public-access defibrillation in Japan. N Engl J Med 2010, 362:994-1004.

19. Kajino K, Kitamura T, Iwami T, Daya M, Ong MEH, Hiraide A, Shimazu T, Kish M, Yamayoshi S: Current termination of resuscitation (TOR) guidelines predict neurologically favorable outcome in Japan. Resuscitation 2013, 84:54-59.

20. Committee ECC: Subcommittees and Task Forces of the American Heart Association: 2005 American Heart Association Guidelines for Cardiopulmonary Resuscitation and Emergency Cardiovascular Care. Circulation 2005, 112:IV1-IV203.

21. Tanigawa $K$, Ito $Y$, Kondo H, Sakamoto T, Shimizu N, Hase N, Hatanaka T, Matsumoto N, Yoshida R, Marukawa S: Emergency Care Activities by ELSTs Based on Japanese Guidelines for Emergency Care and CPR [in Japanese] Available at http://kouroukaken-kyukyusosei.info/wpm/archivepdf/18-20/ 2_02.pdf (accessed 16 November 2012).

22. Jacobs I, Nadkami V, Bahr J, Berg RA, Billi JE, Bossaert L, Cassan P, Coovadia A, D'Este K, Finn J, Halperin H, Handley A, Herlitz J, Hickey R, Idris A, Kloeck W, Larkin GL, Mancini ME, Mason P, Mears G, Monsieurs K, Montgomery W, Morley P, Nichol G, Nolan J, Okada K, Perlman J, Shuster M, Steen PA, Sterz F, International Liaison Committee on Resuscitation; American Heart Association; European Resuscitation Council; Australian Resuscitation Council; New Zealand Resuscitation Council; Heart and Stroke Foundation of Canada; InterAmerican Heart Foundation; Resuscitation Councils of 
Southern Africa; ILCOR Task Force on Cardiac Arrest and Cardiopulmonary Resuscitation Outcomes, et al: Cardiac arrest and cardiopulmonary resuscitation outcome reports: update and simplification of the Utstein templates for resuscitation registries: a statement for healthcare professionals from a task force of the International Liaison Committee on Resuscitation (American Heart Association, European Resuscitation Council, Australian Resuscitation Council, New Zealand Resuscitation Council, Heart and Stroke Foundation of Canada, InterAmerican Heart Foundation, Resuscitation Councils of Southern Africa). Circulation 2004, 110:3385-3397.

23. Schneiderman $L$, Jecker NS, Jonsen AR: Medical futility: its meaning and ethical implications. Ann Intern Med 1990, 112:949-954.

24. Yasunaga $\mathrm{H}$, Miyata $\mathrm{H}$, Horiguch $\mathrm{H}$, Tanabe $\mathrm{S}$, Akahane M, Ogawa T, Koike $\mathrm{S}$, Imamura T: Polulation density, call-response interval, and survival of outof-hospital cardiac arrest. Int I Health Geogr 2011, 10:26.

25. Goto Y, Maeda T, Goto Y: Decision tree model for predicting outcomes after out-of-hospital cardiac arrest in the emergency department. Crit Care 2013, 17:R133.

26. Lippert FK, Raffay V, Georgiou M, Steen PA, Bossaert L: European Resuscitation Council Guidelines for Resuscitation 2010 Section 10. The ethics of resuscitation and end-of-life decisions. Resuscitation 2010, 81:1445-1451.

27. Millin MG, Khandker SR, Malki A: Termination of resuscitation of nontraumatic cardiopulmonary arrest: resource document for the National Association of EMS Physicians position statement. Prehosp Emerg Care 2011, 15:547-554

28. National Association of EMS Physicians and American College of Surgeons Committee on Trauma: Termination of resuscitation for adult traumatic cardiopulmonary arrest. Prehosp Emerg Care 2012, 16:571.

29. Bernard SA, Gray TW, Buist MD, Jones BM, Silvester W, Gutteridge G, Smith $\mathrm{K}$ : Treatment of comatose survivors of out-of-hospital cardiac arrest with induced hypothermia. N Engl J Med 2002, 346:557-563.

30. Kagawa E, Dote K, Kato M, Sasaki S, Nakano Y, Kajikawa M, Higashi A, Itakura K, Sera A, Inoue I, Kawagoe T, Ishihara M, Shimatani Y, Kurisu S: Should we emergently revascularize occluded coronaries for cardiac arrest? Rapidresponse extracorporeal membrane oxygenation and intra-arrest percutaneous coronary intervention. Circulation 2012, 126:1605-1613.

31. Ahn KO, Shin SD, Suh GJ, Cha WC, Song KJ, Kim SJ, Lee EJ, Ong MEH: Epidemiology and outcomes from non-traumatic out-of-hospital cardiac arrest in Korea: a nationwide observational study. Resuscitation 2010, 81:974-981.

32. Ong MEH, Tiah L, Leong BS, Tan EC, Ong VY, Tan EA, Poh BY, Pek PP, Chen $Y$ : A randomised, double-blind, multi-centre trial comparing vasopressin and adrenaline in patients with cardiac arrest presenting to or in the Emergency Department. Resuscitation 2012, 83:953-960.

33. Lin $\mathrm{CH}$, Chiang WC, Ma MH, Wu SY, Tsai MC, Chi CH: Use of automated external defibrillators in patients with traumatic out-of-hospital cardiac arrest. Resuscitation 2013, 84:586-591.

doi:10.1186/cc13058

Cite this article as: Goto et al:: Termination-of-resuscitation rule for emergency department physicians treating out-of-hospital cardiac arrest patients: an observational cohort study. Critical Care 2013 17:R235.

\section{Submit your next manuscript to BioMed Central and take full advantage of:}

- Convenient online submission

- Thorough peer review

- No space constraints or color figure charges

- Immediate publication on acceptance

- Inclusion in PubMed, CAS, Scopus and Google Scholar

- Research which is freely available for redistribution 